\title{
EL ESTILO INGLÉS DE CONTROL PREVENTIVO DE CLÁUSULAS ABUSIVAS*
}

\section{THE ENGLISH WAY FOR PREVENTING UNFAIR TERMS}

\author{
María Elisa MORALES ORTIZ**
}

RESUMEN: Este trabajo plantea que la forma en que el sistema inglés ha implementado el control de cláusulas abusivas es una experiencia que se debería mirar si, haciendo microcomparación, se busca construir una propuesta que mejore nuestro respectivo sistema interno. Diversas razones apoyan esta afirmación. A continuación, se parte explicando por qué resulta recomendable mirar cómo los ingleses han controlado preventivamente cláusulas abusivas y se describe cómo ha funcionado este control aludiendo, además, a algunos de sus resultados. Lo anterior se logra a través de un análisis del trabajo que la Office of Fair Trading realizó en este ámbito. El artículo concluye con una síntesis de las virtudes de este estilo de control preventivo de cláusulas abusivas.

Palabras clave: derecho del consumo, cláusulas abusivas, derecho comparado, derecho inglés, negociación.
ABSTRACT: This paper argues that the way in which the English system has implemented the control of unfair terms is an experience that should be look at if, by making a micro comparison, it is sought to build a proposal that improves our respective internal system. Several reasons support this approach. The following pages explain why it is advisable to look at the preventive control of unfair terms of English law and describe its operation alluding some results. This is achieved through an analysis of the work at the Office of Fair Trading performed in this area. The article concludes with a synthesis of the virtues of this style of preventive control of abusive clauses.

Keywords: Consumer Law, Abusive Clauses, Comparative Law, English Law, Negotiation.

* Artículo recibido el 7 de julio de 2017 y aceptado para su publicación el 5 de febrero de 2019.

** ORCID: 0000-0003-1200-7253. Doctora en derecho por la Universidad de Chile. Abogada, licenciada en ciencias jurídicas y sociales, Universidad Austral de Chile. Académica del Departamento de Ciencias Jurídicas de la Facultad de Ciencias Jurídicas y Empresariales de la Universidad de La Frontera. Miembro del Centro de Investigación sobre Desafíos Internacionales (CIDI), Universidad de La Frontera. Miembro del Centro de Excelencia en Psicología Económica y del Consumo (CEPEC), Universidad de La Frontera. Correo postal: Avenida Francisco Salazar 1145, Temuco, Chile. Correo electrónico: maria.morales@ufrontera.cl. Financiado parcialmente por la Dirección de Investigación de la Universidad de La Frontera, Proyecto DI17-0065 y por la Dirección de Cooperación Internacional de la Universidad de La Frontera, Temuco, Chile.

Boletín Mexicano de Derecho Comparado, nueva serie, año LI, núm. 154, enero-abril de 2019, pp. 171-198.

Esta obra está bajo una Licencia Creative Commons Atribución-NoComercial-SinDerivar 4.0 Internacional, IIJ-UNAM. 
SUMARIO: I. Razones para mirar el estilo inglés de control preventivo de cláusulas abusivas. II. Control preventivo y administrativo de cláusulas abusivas en el derecho inglés. III. Consideraciones finales. IV. Bibliografia.

\section{RAZONES PARA MIRAR EL ESTILO INGLÉS DE CONTROL PREVENTIVO DE CLÁUSULAS ABUSIVAS}

Existe un amplio consenso respecto de que los contratos de adhesión y cláusulas generales deben ser controladas para proteger a los consumidores. Sin embargo, las aproximaciones que los distintos países han dado a este control varían bastante (Appenzzeller, 2017: 60). En Europa, la Directiva 93/13 (Consejo de la Unión Europea, 1993) ${ }^{1}$ permite la implementación tanto de controles llevados a cabo por órganos administrativos como por los tribunales de justicia (control administrativo y judicial, respectivamente). Países con una fuerte tradición pública que han preferido un control de tipo administrativo, como el Reino Unido, han mostrado mejores cifras en la prevención de cláusulas abusivas (Appenzzeller, 2017: 60).

Así, según Micklitz, en el contexto europeo, se distinguen tres modelos de control de cláusulas abusivas, a saber, el modelo alemán, francés e inglés (Micklitz, 2008: 24 y ss.). De estos tres, el sistema inglés ha sido el más exitoso en prevenir la inclusión de cláusulas abusivas en los contratos B2C. ${ }^{2}$ La anterior afirmación se sostiene con base en las siguientes razones.

En primer lugar, el sistema inglés es un ejemplo de recepción favorable de la Directiva 93/13. Así lo concluye Niglia (Niglia, 2003: 184 y ss.) quien, en un estudio sobre la transformación del derecho contractual en Europa, ha visto que el sistema inglés ha reaccionado muy favorablemente a la implementación de la regulación europea contra cláusulas abusivas y, no obstante el Brexit, parece haber consenso en torno a que el derecho del consumo en el Reino Unido se ha visto fuertemente influenciado por el derecho del consumo de la Unión Europea, contenido en directivas (Riefa y Willett, 2018: 673).

1 Consejo de la Unión Europea, 1993, Directiva 93/13/CEE del Consejo, en adelante la Directiva 93/13 o solamente la Directiva.

2 Business to consumer, en castellano la traducción sería empresa a consumidor, relación que hace referencia a un contrato de consumo. Un estudio del control extrajudicial en este tipo de contratos se puede consultar en Nava, 2017: 837-861.

Esta obra está bajo una Licencia Creative Commons

Atribución-NoComercial-SinDerivar 4.0 Internacional, IIJ-UNAM.

Boletín Mexicano de Derecho Comparado, núm. 154, enero-abril de 2019, pp. 171-198. 
Prueba de esta actitud receptiva a la que se alude (Niglia, 2003: 147 y ss.) fue la implementación inicial, prácticamente literal, que en el Reino Unido se hizo de la Directiva ${ }^{3}$ por la Unfair Terms in Consumer Contracts Regulations 1994 (UTCCR). ${ }^{4}$ Incluso, la definición de cláusula abusiva fue incorporada mediante una copia idéntica de la contenida en el artículo 3.1 de la Directiva, no obstante la referencia a la buena $f e,{ }^{5}$ que es un elemento ajeno al common law o que, por lo menos hasta entonces, no había jugado un papel explícito en el derecho contractual inglés. ${ }^{6}$ Aunque en una primera etapa se produjo un problema de superposición normativa, este fue superado con el actual Consumer Rights Act 2015 (CRA) (Morales, 2017). Dicha situación no afectó el control preventivo de cláusulas abusivas, como se verá.

A nivel de aplicación, durante los primeros años de implementación de la Directiva 93/13, el órgano administrativo a cargo de la aplicación de estas disposiciones, en ese entonces el director general of Fair Trading, jugó un rol central en la vigilancia de los contratos de adhesión (Niglia, 2003: 184). Según la Comisión de Comunidades Europeas (Comisión de las Comunidades Europeas, 2000: 26), a lo largo de los cinco primeros años de implementación la Office of Fair Trading (OFT), ${ }^{7}$ en aplicación de la Directi-

3 La Directiva 93/13 fue implementada en el derecho inglés por The Unfair Terms in Consumer Contracts Regulations 1994, posteriormente reemplazada por The Unfair Terms in Consumer Contracts Regulations 1999. Actualmente, la ley que implementa la Directiva es Consumer Rights Act 2015.

4 The Unfair Terms in Consumer Contracts Regulations (UTCCR)1994, 1o. de julio de 1995, modificada por The Unfair Terms in Consumer Contracts Regulations 1999, 1o. de octubre de 1999.

5 Artículo 3.1. de la Directiva: "Las cláusulas contractuales que no se hayan negociado individualmente se considerarán abusivas si, pese a las exigencias de la buena fe, causan en detrimento del consumidor un desequilibrio importante entre los derechos y obligaciones de las partes que se derivan del contrato". Artículo 5.1 del UTCCR 1999: "A contractual term which has not been individually negotiated shall be regarded as unfair if, contrary to the requirement of good faith, it causes a significant imbalance in the parties' rights and obligations arising under the contract, to the detriment of the consumer".

6 Para un análisis de la aplicación de la buena fe como medio de control de cláusulas abusivas en el derecho inglés, véase Micklitz, 2005: 292-429.

7 Desde el 1o. de abril de 2014 este organismo no existe y sus facultades pasaron a diferentes organizaciones incluyendo la Competition and Markets Authority y la Financial Conduct Authority, más información disponible en: https://wwre.gov.uk/government/organisations/ office-of-fair-trading, consultado el 25 de agosto de 2013.

Esta obra está bajo una Licencia Creative Commons Atribución-NoComercial-SinDerivar 4.0 Internacional, IIJ-UNAM. Boletín Mexicano de Derecho Comparado, núm. 154, enero-abril de 2019, pp. 171-198. 
va, examinaba una media de 800 casos por año (Comisión de las Comunidades Europeas, 2000: 34). Esta Comisión creó, además, una base de datos denominada CLAB (cláusulas abusivas) que reunía las sentencias judiciales y las decisiones de órganos administrativos sobre cláusulas abusivas, según la cual desde la expiración del plazo para implementar la Directiva hasta el año 2000, 625 de las 865 acciones administrativas sobre esta materia provenían del Reino Unido (Comisión de las Comunidades Europeas, 2000: 34).

El carácter administrativo del control parece favorecer al sistema inglés sobre el alemán, donde la protección legal del consumidor, en general, tiene un carácter judicial (Cseres, 2005: 160 y 161). En efecto, en Alemania el modelo de control se ha estructurado a propósito de un proceso de "doble colectivización" de la contratación. El primer nivel de colectivización viene dado por el fenómeno de la contratación por adhesión, y el segundo nivel por condiciones generales elaboradas por conjuntos de empresas de un determinado sector. Sobre el primer nivel de colectivización, opera un control judicial ejercido por los tribunales alemanes, empleando principalmente los estándares de bonos mores y buena fe. En control sobre segundo nivel de colectivización se enfoca desde la perspectiva del derecho de la libre competencia (Micklitz, 2005: 25 y 26). Como se sabe, los mecanismo de control administrativo de cláusulas abusivas presentan ciertas ventajas frente al control judicial, ya que este último suele ser más costoso y lento que los primeros (De la Maza, 2012: 119).

En cuanto al ordenamiento francés, ya con la Ley núm. 78-23, de enero de 1978, ${ }^{8}$ Francia contaba con la Commission des Clauses Abusives, cuya función fundamental ha sido emitir recomendaciones, no vinculantes, de supresión o modificación de cláusulas que presenten el carácter de abusivas. ${ }^{9}$ La misma ley consagraba un capítulo a la regulación de las cláusulas abusivas. Una década más tarde, se incorpora a la legislación una acción especial para atacar las cláusulas abusivas. ${ }^{10}$ Luego, esa legislación fue integrada en 1995 en el Code de la Consommation. Aunque este sistema

8 Francia, Loi num. 78-23 du 10 janvier 1978, sobre la protección e información de los consumidores de productos y servicios. Actualmente, artículos L-534-1 del Código de Consumo.

9 Sobre el carácter de las recomendaciones de la Commission des clauses abusives (Libchaber, 1997: 791; Calandri, 2006: 4, y Gumoyar, 2006: 828).

10 Francia, Loi num 88-14 du 5 janvier 1988. Relative aux actions en justice des associations agrees de consommateurs et a l' information des consommateurs.

Esta obra está bajo una Licencia Creative Commons

Atribución-NoComercial-SinDerivar 4.0 Internacional, IIJ-UNAM.

Boletín Mexicano de Derecho Comparado, núm. 154, enero-abril de 2019, pp. 171-198. 
contempla un mecanismo administrativo, las recomendaciones de la Commission des Clauses Abusives no vinculan a los proveedores y, por lo tanto, la única forma de obtener la modificación o supresión de cláusulas es a través del control judicial, con los inconvenientes ya señalados.

De acuerdo con lo dicho en los párrafos anteriores, Niglia destaca el caso inglés por sobre el francés y alemán, justamente debido a la masiva intervención administrativa (Niglia, 2003: 184).

La segunda razón que permite hablar del sistema inglés como un ejemplo es su buen funcionamiento. En palabras de Zwigert y Kötz, dentro de las consideraciones generales del proceso comparativo, si se piensa proponer una solución extranjera, la primera pregunta que habrá que responder es si ha resultado satisfactoria en su país de origen (Zweigert, y Kötz, 1998: 19). Esta pregunta se refiere a la eficacia con que el país elegido ha resuelto el problema (Mancera, 2008: 213-243).

Es muy difícil sostener categóricamente que un sistema de control de cláusulas abusivas es eficaz (Niglia, 2003: 222). Para algunos, medir el grado de eficacia de un sistema de control de cláusulas abusivas es simplemente una tarea imposible (Niglia, 2003: 222).

Por ello es preferible, por lo menos en el contexto de este artículo, hablar de un sistema que funciona o cumple en alguna medida demostrable las funciones que le son propias, construyendo dicha afirmación sobre la base de informes de organismos oficiales y opiniones de la doctrina autorizada en la materia.

El primer antecedente relevante a este respecto es el ya citado informe de la Comisión de Comunidades Europeas (CCE) sobre la aplicación de la Directiva 93/13. ${ }^{11}$ Este documento tuvo por objeto evaluar la aplicación de la norma comunitaria a lo largo de los cinco primeros años transcurridos desde la fecha de vencimiento prevista para su transposición. En efecto, la CCE (Comisión de las Comunidades Europeas, 2000: 26), destaca el caso del Reino Unido y el papel de la, entonces vigente, OFT en la erradicación de las cláusulas abusivas. Esta Oficina iniciaba negociaciones a partir de la recepción de denuncias con el objeto de convencer al respectivo proveedor de que introduzca las modificaciones necesarias a la cláusula en el contrato. Su gestión tuvo gran impacto. Sólo entre 1995

11 No obstante el Brexit, el Reino Unido hasta ahora estuvo obligado a implementar diversas directivas, entre ellas, la 93/13 sobre cláusulas abusivas.

Esta obra está bajo una Licencia Creative Commons Atribución-NoComercial-SinDerivar 4.0 Internacional, IIJ-UNAM. Boletín Mexicano de Derecho Comparado, núm. 154, enero-abril de 2019, pp. 171-198. 
y 1998, 1,200 proveedores modificaron o eliminaron cláusulas estimadas abusivas (Comisión de las Comunidades Europeas, 2000: 26).

$\mathrm{El}$ informe de la CCE que se viene citando, se dio a conocer en el año 2000 siendo este el único reporte que dicho organismo ha emitido sobre la aplicación de la Directiva 93/13 por parte de los Estados miembros (Europe Direct, 2015: 8).

En el caso del Reino Unido, no sólo estudios de la CEE dan información objetiva respecto de su funcionamiento, sino que también reportes a nivel interno. Al respecto se puede citar un estudio de London Economics (London Economics, 2009: 2) cuyos objetivos fueron evaluar la eficacia de un grupo de intervenciones de la OFT donde todos los casos analizados demostraron enormes beneficios para los consumidores en comparación con los costos que dichas medidas implicaron (London Economics, 2009: 2). Los resultados del estudio arrojaron una cifra global anual de $£ 243 \mathrm{mi}$ llones, reflejados como beneficios para los consumidores, frente a un costo de $£ 2,5$ millones para la OFT. La mitad de los grupos de casos estudiados, consistían en la aplicación de la normativa sobre cláusulas abusivas (Europe Direct, 2015: 8).

Este buen funcionamiento del sistema inglés es respaldado, además, por importante doctrina europea especializada en la materia. Un ejemplo de ello es Guido Alpa, quien en un estudio comparado entre la experiencia de implementación de la Directiva 93/13 italiana e inglesa, destaca a esta última como mucho más articulada y eficaz (Alpa, 2008, pp. 75-92). Él mismo, al evaluar el impacto de la Directiva, señala que los italianos miran con muchísimo interés el modelo inglés en el que el trabajo ya realizado por la OFT ha traído resultados impresionantes. ${ }^{12}$

Un par de razones que podrían elevarse en contra de la elección del sistema inglés como objeto de una microcomparación ${ }^{13}$ son: primero, que este ordenamiento pertenece a la tradición del common law, entendido como una particular manera de pensar y aplicar el derecho o como el derecho consagrado en decisiones judiciales, en contraposición al civil law entendido como el derecho principalmente plasmado en leyes o códigos

12 "...italians look with extreme interest to the English model in which the work already carried out by the OFT - now assisted by Financial Services Authority- has obtained impressive results" (Alpa, 2008: 89).

13 Es decir, comparación que aborda un problema legal específico (Zweigert y Kötz, 1998: 6).

Esta obra está bajo una Licencia Creative Commons

Atribución-NoComercial-SinDerivar 4.0 Internacional, IIJ-UNAM.

Boletín Mexicano de Derecho Comparado, núm. 154, enero-abril de 2019, pp. 171-198. 
(David, 1980: 16), lo cual podría dificultar la comparación con sistemas de esta última tradición; y, segundo, el factor cultural que marca importantes límites en los estudios de derecho comparado (Cotterrell, 2006: 711) y que en el caso del Reino Unido resulta bastante particular con relación a sistemas pertenecientes a la tradición romano-germánica.

En cuanto a lo primero, como se ha sostenido en otro lugar:

... a diferencia de lo que pasa con la regulación de los contratos, en general regidos por el common law, la mayoría de la regulación relativa a protección del consumidor en el derecho inglés se encuentra contenida en leyes. Lo anterior se justifica, en parte, por el conflicto entre la idea de protección de la parte débil y las raíces del derecho de contratos conforme a las cuales cada parte debería proteger sus propios intereses al momento de contratar. Otras razones se pueden esgrimir. Por ejemplo, el hecho de que ciertas sanciones que requieren estar establecidas por la ley hayan sido vistas como la única forma para hacer frente a ciertas prácticas comerciales relacionadas con la protección al consumidor. Por otro lado, solo los tribunales superiores están realmente involucrados en el desarrollo del common law y los asuntos de derecho de consumo rara vez llegan a esas instancias (Morales, 2017: 286-287).

Por lo tanto, las características del derecho inglés, como un sistema del common law no pueden ser esgrimidas como un argumento en contra de un posible trasplante, pues el derecho del consumo en este ordenamiento se encuentra regido especialmente por leyes (statutes).

Con relación al factor cultural como un posible obstáculo para la adopción de una solución venida del sistema inglés, este es sólo aparente. En efecto, al tratarse el problema de las cláusulas abusivas un asunto que se ventila a propósito de la contratación, donde los intereses en juego son de carácter patrimonial, se asume que, producto de la globalización económica, las soluciones a estos problemas son de mucho más fácil trasplante que soluciones a asuntos con contenido extra patrimonial como lo son los relativos al derecho de familia o el derecho sucesorio, escenarios donde las sociedades son más diversas y más resistentes a la recepción (Graziedei, n.d: 472).

No obstante lo anterior, para una verdadera adaptación legislativa del derecho interno de la solución inglesa, más allá de una simple comparación teórica, un estudio interdisciplinario que se preocupe, además, de 
estudiar los intereses en juego y la viabilidad cultural, se estima imprescindible.

Con todo, los propósitos de este trabajo son mucho más modestos. Sólo se pretende exponer una solución que ha demostrado, dentro de su contexto, lograr prevenir la inclusión de cláusulas abusivas en los contratos $\mathrm{B} 2 \mathrm{C}$ a un gran número de contratos y que se estima debe ser tenida en cuenta como un ejemplo si se pretende mejorar la experiencia interna.

\section{CONTROL PREVENTIVO Y ADMINISTRATIVO DE GLÁUSULAS ABUSIVAS EN EL DERECHO INGLÉS}

\section{1. Órganos administrativos encargados del control preventivo de cláusulas abusivas}

Desde la implementación de la Directiva 93/13, se han sucedido tres organismos a cargo del control de cláusulas abusivas en el Reino Unido. Desde 1973 a 2002 el Director General of Fair Trading (DGFT); desde 2002 a 2014 la Office of Fair Trading (OFT) y, desde 2014 a la fecha, la Competition Markey Authority (CMA).

Este artículo se centra en la OFT, sin embargo, se hace referencia al DGFT como antecedente y a CMA como continuadora.

\section{A. Director General of Fair Trading}

El control preventivo fue implementado en el derecho inglés, en primera instancia, por la UTCGR 1994, donde se imponía el deber al $\mathrm{DGFT}^{14}$ de investigar las denuncias relativas a cláusulas abusivas y ejercer sus facultades administrativas de aplicación del derecho del consumo o accionar frente a los tribunales, en caso de ser necesario, para obtener una orden judicial (injunction). Entonces, y hasta ahora, sólo se puede determinar judicialmente la abusividad de una cláusula.

De conformidad a la Fair Trading Act (FTA, 1973), frente a una conducta perjudicial o injusta para los consumidores por parte de una empresa, el DGFT estaba facultado para comunicarse con sus representantes para obtener una garantía escrita de que se abstendría de ejecutar la

14 Creado por el Fair Trading Act 1973 el 25 de julio de 1973.

Esta obra está bajo una Licencia Creative Commons

Atribución-NoComercial-SinDerivar 4.0 Internacional, IIJ-UNAM.

Boletín Mexicano de Derecho Comparado, núm. 154, enero-abril de 2019, pp. 171-198. 
respectiva conducta (undertaking). En caso de que la empresa no cumpliera con lo comprometido, el DGFT quedaba facultado para accionar ante los tribunales. ${ }^{15}$ Sin embargo, la acción judicial fue, y ha sido, vista como último recurso (Bright, 2000: 334).

El trabajo del DGFT se centró más bien en acciones extrajudiciales (undetakings). En efecto, el DGFT se preocupó de perseguir enérgicamente las denuncias sobre cláusulas abusivas logrando la alteración de contratos en varios sectores empresariales a través de la negociación. La gran mayoría de los casos se resolvían por medio de esta vía. Bright describe muy bien este modo de operar:

El Reino Unido estaba muy por detrás de otros Estados miembros en el control de cláusulas abusivas antes de la Directiva. Había, sencillamente, que ponerse al día. Pero también está claro que la forma seleccionada para la aplicación de las normas ha resultado particularmente eficaz. En virtud de UTCGR, el DGFT tiene el deber de investigar las denuncias sobre cláusulas abusivas, descrito por él como "la extensión más importante de mis responsabilidades en materia de protección de los consumidores desde los años setenta”. La Unidad de Cláusulas Abusivas ha perseguido enérgicamente las denuncias y ha asegurado la modificación de contratos en varios sectores empresariales a través de un proceso de negociaciones rigurosas, casi sin necesidad de recurrir a acciones judiciales. ${ }^{16}$

15 Secciones 34 y 35. El caso más conocido es DGFT v First National Bank. DGFT v First National Bank [2001] UKHL 52. Esta fue la primera vez que la Cámara de los Lores (the House of Lords) evaluó la abusividad de una cláusula contractual bajo UTCCR 1994. Se cuestionó una cláusula contenida en un contrato de préstamo bancario, que permitía al banco cargar al consumidor la tasa de interés convenida sobre el monto de capital pendiente y cualquier interés devengado hasta la fecha del juicio. Mediante esta cláusula el banco se aseguraba de que en caso de incumplimiento el interés se calcularía de acuerdo con la tasa contractual y no de acuerdo con la tasa normal más baja aplicable para juicios de este tipo. Frente a esto, el DGFT, haciendo uso de sus facultades, accionó ante los tribunales para impedir que esta cláusula se siguiera utilizando. El banco argumentó que esta era de aquellas cláusulas excluidas de la prueba de abusividad. En apelación, la Cámara de los Lores decidió por unanimidad que la cláusula no era abusiva. Para ver un análisis del caso, véase MacDonald, 2002: 763-773.

16 "The United Kingdom was some way behind other member states in controlling the use of unfair contract terms prior to the Directive. There was, quite simply, more catching up to be done. But it is also clear that the manner selected for enforcement of the Regulations has proven particularly effective. Under the 1994 Regulations, the Director General of Fair Trading is given a duty to investigate complaints about unfair terms, des- 
Sus investigaciones no se acotaban exclusivamente a contratos particulares, sino que eran más bien sectoriales, extendiéndose a asociaciones de proveedores con el objeto de expandir los efectos de la negociación, siguiendo así el ya mencionado modelo de enforcement piramidal, modelo que tanto OFT y como CMA han mantenido, en gran parte por haber demostrado tener un muy buen funcionamiento. De hecho, siguiendo este modelo, entre 1995 y 1998, 1.200 empresas modificaron o eliminaron de sus contratos ciertas cláusulas estimadas abusivas (Comisión de las Comunidades Europeas, 2000: 26).

Luego, y como ya se ha dicho, UTCCR 1994 fue remplazada por UTCGR 1999. No hubo cambios sustanciales. Se buscó dar cabal cumplimiento al artículo 7o. de la Directiva 93/13 que permite a las organizaciones con interés en la protección de los consumidores, acudir a los órganos judiciales o administrativos competentes con el fin de que éstos determinen si ciertas cláusulas contractuales, redactadas con vistas a su utilización general, tienen carácter abusivo, y apliquen los medios adecuados y eficaces para que cese la aplicación de dichas cláusulas. Pues bien, hasta ese entonces, sólo el DGFT podría accionar frente a los tribunales por dicho motivo.

Así, UTCGR 1999 vino a extender a otros organismos la facultad de accionar frente a cláusulas consideradas abusivas (enforcement bodies o public qualifying bodies). Además, entre otros cambios, se dio facultad a los qualifying bodies para requerir documentos, tales como los contratos donde consten las cláusulas generales, y la información que fuera necesaria con el objeto de aplicar la ley. En todo caso, estos organismos debían notificar al DGFT tanto de los acuerdos o compromisos a que hayan arribado con las empresas como de los procedimientos judiciales que hayan iniciado, pudiendo el director publicar esta información.

En 2002, la Enterprise Act (EA 2002) suprimió la figura del DGFT transfiriendo todas sus funciones a la OFT.

cribed by him as 'the most important extension of my consumer protection responsibilities since the 1970s'. The Unfair Contract Terms Unit established in response has energetically pursued complaints and has been able to secure the alteration of contracts in several business sectors through a process of rigorous negotiations, with almost no need to resort to legal actions" (Bright, 2000: 332). La traducción es propia.

Esta obra está bajo una Licencia Creative Commons

Atribución-NoComercial-SinDerivar 4.0 Internacional, IIJ-UNAM.

Boletín Mexicano de Derecho Comparado, núm. 154, enero-abril de 2019, pp. 171-198. 


\section{B. Office of Fair Trading}

La OFT fue durante más de cuarenta años ${ }^{17}$ el más importante órgano responsable de proteger los derechos de los consumidores ${ }^{18}$ en el Reino Unido. Su principal función era velar porque los mercados funcionen bien para los consumidores. ${ }^{19}$

Dentro de sus facultades se encontraban informar a los consumidores, actuar como un organismo coordinador, mantener bajo revisión las prácticas de las empresas para que sean justas y competitivas, fomentar los códigos de buenas prácticas, además de una serie de específicas facultades para enfrentar a los proveedores que infringen el derecho del consumo, entre ellas, el control de las cláusulas abusivas (Howells y Weatherill, 2005: 574-575).

Aunque la OFT no era el órgano encargado de velar por la libre competencia, ${ }^{20}$ ya que esta función correspondía a la Competition Commission $(\mathrm{CG})$, sus funciones reconocían la íntima conexión entre la protección del consumidor y la libre competencia, y ambas agencias se encontraban vinculadas legalmente bajo un esquema colaborativo. En efecto, era deber de la OFT remitir a la CG ciertas fusiones o situaciones de mercado para que la última iniciara una investigación al respecto y tomara las medidas que procedían. ${ }^{21}$ En todo caso, este es un asunto que se relaciona más con otros derechos del consumidor, como la libre elección del bien o servicio que puede verse afectado, por ejemplo, cuando en el mercado hay monopolios o colusiones.

En cuanto al deber de informar y educar a los consumidores, en el específico ámbito de las cláusulas abusivas, la OFT publicaba detallada-

17 Se dice que más de cuarenta años entendido a la figura del DGFT dentro del departamento de la OFT (1973-2014).

18 Además de los mecanismos ejercidos por la OFT, hay muchos otros dirigidos a proteger a los consumidores, tales como diversas instancias de ADR, el ombudsman, etcétera. Este trabajo se enfoca en la OFT, para ver otros mecanismos consúltese Woodroffe y Lowe, 2013: 213 y ss.

19 Excede de los objetivos de este trabajo analizar las ventajas y desventajas de esta naturaleza dual. Para ello véase Howells y Weatherill, 2005: 574.

20 El órgano encargado de garantizar una sana competencia entre las empresas en el Reino Unido para el beneficio final de los consumidores y la economía era la Competition Commission.

21 De acuerdo con EA, 2002. 
mente los compromisos de las empresas y las órdenes judiciales recaídas en procedimientos sobre cláusulas abusivas (UTCGR, Reg. 15, 1999).

En la práctica, la difusión se ha llevado a cabo mediante boletines periódicos que contenían informes pormenorizados de los casos, ${ }^{22}$ junto con comunicados de prensa relativos a los "triunfos" significativos de la agencia (Bright, 2000: 337). Según la OFT, la publicación periódica de su trabajo permitía, además, la coordinación con los demás enforcers (organismos reguladores que aplican el derecho del consumo), facilitar las denuncias sobre nuevos incumplimientos y educar a otras empresas. ${ }^{23}$

Conforme a lo anterior, se puede ver aquí una forma de control administrativo porque lo que busca es, además de informar a los consumidores, dar a conocer a los proveedores cuáles son las cláusulas que son consideradas abusivas, con el objeto de que se abstengan de utilizarlas. Sería entonces, de un lado, un mecanismo de control administrativo preventivo y, voluntario del otro, si se logra el efecto de abstención espontánea por parte del proveedor. Según la OFT:

La publicación de nuestro trabajo nos permite promover la consciencia de los consumidores sobre sus derechos, así como permitir que otros enforcers busquen fácilmente en la página web recientes o actuales acciones que la OFT u otras agencias puedan estar llevando a cabo.

Esto ayuda a prevenir múltiples criterios con respecto a la empresa por parte de diferentes enforcers, así como a promover la coherencia del enfoque, compartiendo tales resultados y otros detalles del caso con otros agentes aplicadores del derecho. ${ }^{24}$

22 En su última etapa, la Office of Fair Trading dejó de publicar boletines, pero produjo informes anuales de su trabajo. Actualmente es posible revisar los casos detalladamente, tanto undertakings como court actions, disponibles en: http://webarchive.nationalarchives. gov.uk/20140525130048/http://oft.gov.uk/OFTwork/consumer-enforcement, consultado el 3 de enero de 2017; los reportes anuales, desde 2000 a 2013, disponibles en: http://webarchive. nationalarchives.gov.uk/20140402142426/http:/www.oft.gov.uk/OFTwork/publications/publication-categories/corporate/annual-report, consultado el 4 de enero de 2017; y todos los boletines y guías, disponibles en: http://webarchive.nationalarchives.gov.uk/20140525130048/http:// wrwre.oft.gov.uk/OFTwork/publications/publication-categories/guidance/unfair-terms-consumer, consultado el 4 de enero de 2017.

23 OFT, The Retention of Undertakings on the Consumer Regulations Website, disponible en: http://webarchive.nationalarchives.gov.uk/20140525130048/http://oft.gov.uk/ crw/445520/630922/retention_policy, consultado el 21 de diciembre de 2016.

24 "Publicising our work allows us to promote consumers" awareness of their rights, as well as allowing other enforcers easily to search the website for recent or current action

Esta obra está bajo una Licencia Creative Commons

Atribución-NoComercial-SinDerivar 4.0 Internacional, IIJ-UNAM.

Boletín Mexicano de Derecho Comparado, núm. 154, enero-abril de 2019, pp. 171-198. 
Así, la OFT utilizó la divulgación de sus resultados para producir un efecto de coordinación natural entre las agencias (coordinación horizontal), proveedores y consumidores (vertical). Lo anterior refuerza el rol coordinador expresamente asignado. Se generaba de esta forma una especie de advertencia a los proveedores y una alerta a los consumidores respecto de qué cláusulas, concretamente, estaban siendo consideradas abusivas.

Por otro lado, la OFT tuvo la misión ${ }^{25}$ de alentar a las asociaciones de empresarios o comerciantes a redactar códigos de buenas prácticas que salvaguarden y promuevan los intereses de los consumidores, proveyendo de un sello o símbolo oficial a aquellos códigos aprobados (EA, Section 8). Bajo esta fórmula, las asociaciones tenían el deber de realizar un informe anual a través del cual la OFT monitoreaba el cumplimiento del código.

En 2001, la OFT robusteció el sistema elevando las exigencias de aprobación de códigos de buenas prácticas. En 2006 se evaluó el nuevo sistema y resultó ser bastante eficiente. Los costos de implementación resultaron ser más bajos que los beneficios obtenidos y el número de denuncias en contra de los asociados resultó ser más bajo en comparación a proveedores fuera del esquema. Por otro lado, las asociaciones de proveedores adscritas mejoraron su reputación y aumentaron el número de solicitudes de membresía (Ramsay, 2012: 288-292).

Ahora, este sistema favorece el cumplimiento de las normas de protección de los consumidores consideradas generalmente, y de ahí puede ser considerado un mecanismo de control de cláusulas abusivas. En esa perspectiva, se trataría de una forma mixta de control de cláusulas abusivas, que combina el control administrativo estatal con la voluntariedad, ya que el sometimiento del respectivo código a la aprobación de la OFT es voluntario para los proveedores.

En cuanto a la función especialmente dirigida al control de cláusulas abusivas, como ya se sabe, con la modificación a UTCGR en 1999 se ra-

which the OFT or other designated enforcers may be taking forward. This helps to prevent multiple approaches to business by different enforcers, as well as promoting consistency of approach by sharing such outcomes and other case details with other enforcers." OFT. The retention of Undertakings on the Consumer Regulations Website, disponible en: http:// webarchive.nationalarchives.gov.uk/20140525130048/http://oft.gov.uk/crw/445520/630922/retention_policy, consultado el 21 de diciembre de 2016. La traducción y cursivas son propias.

25 Desde 2013 esta facultad fue transferida al Trading Standards Institute. 
dicó formalmente en varios organismos, además de la OFT. Se trata de algunos reguladores sectoriales y asociaciones de consumidores que, en su conjunto, han sido denominados qualifying bodies (también denominados enforcers, sólo en el caso de los reguladores), por ejemplo: The Data Protection Registrar, The Director General of Electricity Supply, The Director General of Gas Supply, The Director General of Electricity Supply for Northern Ireland, The Director General of Gas for Northern Ireland, The Director General of Telecommunications, The Director General of Water Services, The Rail Regulator, Every Weights and Measures Authority in Great Britain, The Department of Economic Development in Northern Ireland, y Consumers' Association. ${ }^{26}$

Para evitar la superposición de facultades o la duplicación de procedimientos, la UTCGR previó la coordinación entre OFT y los demás reguladores. ${ }^{27}$ De hecho, los qualifying bodies se encontraban facultados para aceptar compromisos de las empresas o accionar judicialmente en caso de estimar que una cláusula era abusiva, pero con la limitación de notificar siempre a la OFT con, por lo menos, catorce días de anticipación (UTCCR, Regulation 12[2]). Adicionalmente, estos debían informar a dicha agencia de todos los compromisos o undertakings y sobre el resultado de los juicios (UTCCR, Regulation 14). Esta coordinación fue fortalecida mediante diferentes acuerdos alcanzados entre la OFT y los qualifying bodies (Howells y Weatherill, 2005: 294).

El escenario recién descrito ha sido calificado por Howells y Weatherill como un "reinvigorated pattern of enforcenment" (Howells y Weatherill, 2005: 294) o modelo revigorizado de control de cláusulas abusivas que da énfasis al control por órganos públicos, lo cual parece bastante adecuado toda vez que asegura la exclusión de la asimetría entre las partes.

\section{Competition and Markets Authority}

La CMA fue establecida por la Enterprise and Regulatory Reform Act (ERRA, 2013). Actualmente, es el principal organismo encargado de promover la libre competencia en beneficio de los consumidores en el Reino Unido. Fue establecida en el contexto de una serie de reformas que

26 Schedule 1, Regulation 3, Part One and Two, UTCGR.

27 El rol coordinador de la OFT también está previsto en la sección 214 de la EA.

Esta obra está bajo una Licencia Creative Commons

Atribución-NoComercial-SinDerivar 4.0 Internacional, IIJ-UNAM.

Boletín Mexicano de Derecho Comparado, núm. 154, enero-abril de 2019, pp. 171-198. 
buscaban fortalecer la protección de los consumidores y la libre competencia en beneficio de la economía y de los intereses de los consumidores. ${ }^{28}$ Cuando la OFT fue suprimida, el 1o. de abril de $2014,{ }^{29}$ sus funciones pasaron a distintos órganos. Entre ellos la Financial Conduct Authority (FCA) y CMA.

La función de control de cláusulas abusivas fue transferida a $\mathrm{CMA}^{30}$ con facultades muy similares a la OFT.

Entre sus funciones, al igual que la OFT, tiene el deber de informar y educar; es un órgano coordinador respecto de los demás regulators, ${ }^{31}$ tiene la facultad de considerar las denuncias, pero, en este caso, continuando con el enfoque sectorial con el que venía trabajando la OFT, cuando aquellas infracciones se relacionen con fallas sistemáticas del mercado; ${ }^{32}$ también debe monitorear los compromisos dados por las empresas; accionar ante los tribunales en caso de incumplimiento; etcétera.

A diferencia de la OFT, el foco de CMA es el buen funcionamiento del mercado, y en el campo de la protección del consumidor sus facultades

28 Esta medida formó parte de varias reformas que tuvieron por objeto proporcionar una protección más fuerte para los consumidores y promover la libre competencia en beneficio de la economía de los consumidores (OFT, 2014:17).

29 Según el profesor Stephen Weatherill, las razones de este cambio son, principalmente, presupuestarias, además de propender a la mejor protección de los intereses de los consumidores. Reunión con Stephen Weatherill el 28 de mayo de 2015 en Somerville College, Oxford University, Reino Unido.

30 CMA tomó sobre sí algunas de las facultades de la OFT, específicamente las facultades de control de cláusulas abusivas. Además, es continuadora de la Competition Commission.

31 "(1) In this Schedule «regulator» means - (a) the CMA, (b) the Department of Enterprise, Trade and Investment in Northern Ireland, (c) a local weights and measures authority in Great Britain, (d) the Financial Conduct Authority, (e) the Office of Communications, (f) the Information Commissioner, (g) the Gas and Electricity Markets Authority, (h) the Water Services Regulation Authority, (i) the Office of Rail Regulation, (j) the Northern Ireland Authority for Utility Regulation, or (k) the Consumers' Association", Shedule 3, CRA, 2015. Esta coordinación sólo rige en materia de cláusulas abusivas, porque existe otro órgano coordinador general llamado Consumer Protection Partnership.

32 Esto se refiere a casos que afectan a sectores del mercado o a varias empresas más que a empresas individualmente consideradas, a menos que esto último genere un impacto en el mercado en su conjunto. Gordana Cumming, 2 de junio de 2015.Gordana.Cumming@ cma.gsi.gov.uk (en línea) Re: Asking for meeting (CMA's approach to unfair terms), consulta: 5 de enero de 2017. Véase CMA (2014: 8). 
se limitan al control de cláusulas abusivas ${ }^{33} \sin$ embargo, con muy similares competencias en dicho ámbito.

Como se ha dicho, este trabajo se enfoca en revisar y analizar el control preventivo de cláusulas abusivas a manos de OFT. Las razones de dicha elección son, primero, porque la OFT ejerció esta función durante quince años, aproximadamente, y CMA aún no cumple dos años en dicho rol, razón por la que no es viable ni justo una verdadera evaluación de su desempeño, y segundo, porque no existen importantes diferencias en cuanto a la forma y facultades de ejercer el control. El estilo es el mismo.

\section{Forma en que opera el control preventivo de cláusulas} abusivas en el derecho inglés ${ }^{34}$

Las facultades para controlar cláusulas abusivas de la OFT se encontraban en la UTCGR y en la parte 8 de la EA referida a la aplicación del derecho del consumo.

La OFT y los qualifying bodies tenían el deber de considerar las denuncias sobre cláusulas abusivas en contratos con condiciones generales (contract term drawn up for general use), salvo que estas sean infundadas o temerarias. El control se podía ejercer respecto de cláusulas que se estuviesen utilizando en un contrato - control represivo - o que estén siendo recomendadas para ser utilizadas — control preventivo-. ${ }^{35}$

Si un qualifying body había decidido conocer, estaba obligado a notificar de ello a la OFT y ésta se abstenía de actuar en el mismo caso, ${ }^{36}$ justamente para evitar la duplicación de procedimientos.

Ante una denuncia, tanto la OFT como los qualifying bodies, tenían más de un camino: instar el cumplimiento voluntario, ejercer sus facultades de control administrativo, o accionar ante los tribunales. En todo caso, debían fundamentar su decisión de entablar o no una acción judicial en contra de

33 Las demás facultades de aplicación del derecho del consumo pasaron al Trading Standard Service.

34 Un trabajo que describe el control preventivo de cláusulas abusivas en el derecho inglés y que lo compara con el derecho chileno, se puede ver en Morales (2017: 67-92).

35 Véase sección 10 y ss. de la EA.

36 Salvo que se trate de denuncias infundadas o temerarias o si otro enforcer notificó a la OFT que ha aceptado conocer de la denuncia (UTCGR, Regulation 14, 1999).

Esta obra está bajo una Licencia Creative Commons

Atribución-NoComercial-SinDerivar 4.0 Internacional, IIJ-UNAM.

Boletín Mexicano de Derecho Comparado, núm. 154, enero-abril de 2019, pp. 171-198. 
quien aparezca utilizando o recomendando utilizar una cláusula estimada abusiva. ${ }^{37}$

En caso de llegar al ejercicio del control judicial esta era, y sigue siendo, la única vía para que la cláusula sea declarada abusiva, y el efecto es que dicho término no vinculará a los consumidores. ${ }^{38}$ Una orden judicial podía pronunciarse no sólo sobre la abusividad de una cláusula general determinada, sino que además se podía extender a cualquier cláusula similar o con el mismo efecto, que esté siendo utilizada o esté siendo recomendada para ser utilizada (UTCGR, section 12).

Es importante aclarar que la OFT no estaba facultada para representar el interés individual de los consumidores, ${ }^{39}$ pero esto no excluía el derecho de los particulares para entablar acciones en contra de los proveedores en forma independiente de la acción de cualquier organismo.

Tanto la OFT como los demás enforcers, tenían la facultad de requerir documentos e información para evaluar las denuncias y/o verificar si se ha cumplido o no con los compromisos u órdenes judiciales.

La OFT, además, debía publicar detalles sobre los compromisos y las órdenes judiciales dadas bajo UTCGR e informar a cualquier persona que lo requiera si una cláusula había sido objeto de alguna acción administrativa $u$ orden judicial. ${ }^{40}$

37 En esta decisión se tomaba en cuenta los compromisos o undertakings (Woodroffe y Lowe, 2013: 200).

38 La norma fue copiada de la Directiva 93/13. Respecto al alcance de esta sanción "[d] ebe señalarse que el legislador de la Unión no ha ido más lejos en la determinación de la sanción aplicable a las cláusulas abusivas y, en particular, del modo en que los Estados miembros deben disponer que éstas no surtan efectos vinculantes, tal como se exige en el artículo 6o., apartado 1, de la Directiva 93/13. La utilización del futuro de indicativo («no vincularán») nada revela en cuanto a la posible intención de ese legislador de dotar a la falta de efecto vinculante de una dimensión retroactiva. Ese mismo legislador ha optado claramente por no emplear un término jurídico más preciso como hubiera sido el caso, por ejemplo, de una referencia expresa a la nulidad, a la anulación o a la resolución. La expresión utilizada es efectivamente neutra, como ya señalaba el abogado general Trstenjak en sus conclusiones presentadas en el asunto Invitel". Conclusiones del abogado general Sr. P. Mengozzi, presentadas el 13 de julio de 2016. ECLI identifier: ECLI: EU: C:2016:552.

39 Los consumidores pueden dirigir sus denuncias a la Citizens Advice Consumers Service.

40 Actualmente es posible revisar los casos detalladamente, tanto undertakings como court actions, en: http://webarchive.nationalarchives.gov.uk/20140525130048/http://oft.gov.uk/ OFTwork/consumer-enforcement, consultado el 3 de enero de 2017.

Esta obra está bajo una Licencia Creative Commons Atribución-NoComercial-SinDerivar 4.0 Internacional, IIJ-UNAM. Boletín Mexicano de Derecho Comparado, núm. 154, enero-abril de 2019, pp. 171-198. 
En resumen, si la OFT consideraba que una denuncia contenía una potencial cláusula abusiva, podía adoptar alguna de las siguientes estrategias (Woodroffe y Lowe, 2013: 202):

a) Abrir un diálogo con el proveedor, invitándolo a modificar o eliminar la cláusula que, en su opinión, era abusiva.

b) Si lo anterior fracasaba, se buscaba llegar a un compromiso o undertaking. ${ }^{41}$

c) Como último recurso, se podía accionar judicialmente.

En ese esquema es posible identificar tres formas diferentes de aplicación de la norma o enforcement remedies (Ramsay, 2012: 220 y 221): 1) acuerdos informales logrados a través de negociación; 2) órdenes administrativas, dentro de las cuales encontramos undertakings o compromisos de cumplimiento voluntario, y 3) órdenes judiciales.

La negociación informal, como primera etapa, no encontraba fuente detallada en la ley, sino que se basaba en las facultades amplias de la OFT de proteger a los consumidores del uso de cláusulas abusivas. Es decir, se trataba de un mecanismo desregulado que buscaba el cumplimiento voluntario. Una negociación podía o no terminar en un compromiso. Un compromiso o undertaking, viene precedido de una negociación que tiene por objeto rectificar o prevenir algún problema específico (Ramsay, 2012: 253), en este caso el uso de cláusulas abusivas.

La diferencia entre la negociación informal y el compromiso o undertaking, es que lo primero es amplio, informal, y no está regulado. En cambio, un undertaking es un remedio administrativo con base legal, donde una un sujeto se compromete ante el órgano administrativo respecto de hacer o dejar de hacer algo específico de acuerdo con la legalidad, y se conecta con la negociación porque en una primera etapa comporta una negociación face-to-face. Luego, el compromiso al que se ha arriba es monitoreable y exigible en el sentido que, si no es cumplido, puede la OFT entablar una acción judicial.

La negociación era el primer paso. Cuando el proveedor pertenecía a una asociación, se tomaba contacto con ésta, igualmente para un ma-

41 Contemplado, además, en Enterprise Act (2002: section 219). Se trata de compromisos ofrecidos por el infractor y pueden incluir el cese de una conducta, compensaciones, cumplir con algún programa, etcétera (Ramsay, 2012: 253).

Esta obra está bajo una Licencia Creative Commons

Atribución-NoComercial-SinDerivar 4.0 Internacional, IIJ-UNAM.

Boletín Mexicano de Derecho Comparado, núm. 154, enero-abril de 2019, pp. 171-198. 
yor impacto. ${ }^{42}$ Un ejemplo de lo anterior es el famoso caso de la OFT y la British Vehicle Rental and Leasing Association donde, a través de negociación se llegó a establecer un contrato tipo para el sector, con gran impacto, ya que la asociación representaba por sí sola un $85 \%$ del sector de arrendamiento de automóviles en el Reino Unido y, al recaer el acuerdo sobre condiciones generales de la contratación, se concretó un potente control preventivo de cláusulas abusivas.

La OFT tenía el deber de monitorear el cumplimiento (Enterprise, Act 2002: Section 92) de estos acuerdos; y frente a un incumplimiento, el próximo paso era accionar judicialmente. No obstante la posibilidad de activar el control judicial, la agencia continuó en la misma línea que el DGFT considerando esta posibilidad como última ratio. Se buscaba persuadir a los proveedores para que abandonen o modifiquen las cláusulas consideradas abusivas dejando, en todo caso, abierta la vía judicial para el incumplimiento del compromiso alcanzado (undertaking). Esta fórmula, induce el cumplimiento de la norma sin necesidad de tomar formalmente acciones judiciales (Howells y Weatherill, 2005: 293).

Otro ejemplo de aplicación de un undertaking es el caso Carcraft Automotive Group Limited..$^{43}$ A partir de una serie de denuncias de los consumidores, la OFT decidió investigar a este proveedor y a otras empresas asociadas a él, ${ }^{44}$ descubriendo una serie de infracciones, entre ellas a UTCGR. La agencia estimaba que Carcraft contemplaba cláusulas potencialmente abusivas en los términos y condiciones de su contrato de posventa. La agencia advirtió a Carcraft que consideraría la posibilidad de accionar judicialmente, en caso de que no se acordasen compromisos satisfactorios. Finalmente, la empresa realizó los cambios necesarios y ofreció un compromiso a la OFT.

De acuerdo con los undertakings dados, la empresa se comprometió, entre otras cosas, a no utilizar cláusulas que tengan como efecto: imponer responsabilidad a los consumidores de revisar el historial del vehículo an-

42 OFT, 1996, Unfair Contract Terms Bulletin, núm. 1.

43 CG Automotive Group Limited: Investigation into alleged unfair practices by a national second hand car dealer Case Reference: Carcraft - CRE-E//25462. Case opened: may 2009. Case closed: october 2011. Disponible en: http://webarchive.nationalarchives.gov. uk/20140525130048/http://wrewreoft.gov.uk/OFTwork/consumer-enforcement/consumer-enforcement-completed/cc-automotive, consultado el 21 de enero de 2015.

44 All in One Finance Limited, UK Car Group Limited, Pennine Metals A Limited y Pennine Metals C Limited. 
tes de la venta; negar el kilometraje de los vehículos; y, dar a la empresa absoluta discreción en cuanto a otorgar garantía o no.

Según Woodroffe y Lowe (2013: 202), es aquí donde radica el gran impacto que tuvo la introducción de la UTCGR que fue medido no por el volumen de litigios, sino por la cantidad de actividad extrajudicial de aplicación de la normativa, y sobre esa base se sostiene que desde entonces los consumidores han estado en mejor posición que antes. De hecho, aunque muy pocos casos llegaban a los tribunales, miles de cláusulas eran revisadas por la OFT. Los autores dan como ejemplo lo informado en los boletines 21 y 22 de la OFT, los cuales revelan que entre los meses de julio y diciembre de 2002, aproximadamente 765 cláusulas fueron modificadas o suprimidas como resultado del trabajo de la OFT y unas 38 bajo el trabajo de lo qualifying bodies. Además, las negociaciones de la OFT han resultado económicamente eficientes, ahorrando cientos de millones de libras en costos de litigio (Scottish Law Commission, 2012: 21). ${ }^{45}$

Siempre dentro de un modelo de pyramid enforcement, el trabajo de la OFT fue evolucionando (Ramsay, 2012: 317 y 22). En una primera etapa (hasta 2002) sólo había accionado judicialmente una vez (OFT vs. First $\mathcal{N a -}$ tional Bank) privilegiando notoriamente las negociaciones llegando a destacar dentro del contexto europeo por la basta acción administrativa llevada a cabo. ${ }^{46}$ A partir de 2002 es posible mencionar algunos casos judiciales. ${ }^{47}$

El control judicial que se ejercía a instancia de la OFT era un control judicial preventivo y su impacto era igualmente importante no sólo porque se ejercía sobre condiciones generales, sino que también por la fuerza del precedente que, indirectamente, disuadía el uso de cláusulas similares a las

\footnotetext{
45 La traducción es propia.

46 "La base CLAB revela la importancia de los casos tratados en el Reino Unido por vía administrativa desde la expiración del plazo previsto para la transposición de la Directiva: 625 de las 865 acciones administrativas registradas hasta ahora en la base de datos proceden de ese Estado miembro. La Comisión puso en marcha el proyecto «CLAB» (cláusulas abusivas) inmediatamente después de la adopción de la Directiva 93/13/CEE. Se trataba de crear una herramienta que permitiera dar seguimiento a la aplicación práctica de la Directiva por medio de la creación de una base de datos que reuniera las «jurisprudencias nacionales» en materia de cláusulas abusivas" (Comisión de las Comunidades Europeas, 2000: 12 y 34).

47 Entre ellos: Office of Fair Trading v Abbey National plc and Others [2009] UKSC 6; Office of Fair Trading v Foxtons Ltd [2009] EWHC 1681 (Ch); The Office of Fair Trading v Ashbourne Management. Services Ltd छors [2011] EWHC 1237 (Ch) (Ramsay, 2012: 326 y ss).
}

Esta obra está bajo una Licencia Creative Commons

Atribución-NoComercial-SinDerivar 4.0 Internacional, IIJ-UNAM.

Boletin Mexicano de Derecho Comparado, núm. 154, enero-abril de 2019, pp. 171-198. 
que ya se habían declarado judicialmente abusivas. Por ejemplo, en OFT v Ashbourne Management Services $L t d .{ }^{48}$ la empresa demandada actuó en representación de 700 gimnasios del Reino Unido, cuyos contratos administraba, reclutando para ellos alrededor de 300 mil clientes, pactando las mismas condiciones generales. En este caso, la High Court declaró que varias de esas cláusulas eran abusivas y por lo tanto no vinculantes para los consumidores. A partir de esa decisión la OFT notificó a todos los gimnasios para que revisaran sus contratos de adhesión y verificaran si estos contenían cláusulas similares; abrió investigaciones, y trabajó en conjunto con las empresas en la mejora de los contratos, firmando varios acuerdos con los proveedores. ${ }^{49}$

Dentro de su evolución, en una segunda etapa (desde el año 2000, aproximadamente), la OFT comenzó a concentrar su trabajo en "highimpact cases" (Ramsay, 2012: 326) que involucraban grandes grupos empresariales o asociaciones de comerciantes y super complaints. ${ }^{50}$

Como consecuencia de este enfoque sectorial (sector-wide approach) la cantidad de casos revisados por la OFT disminuyeron en cantidad, pero sin afectar el alcance de su intervención. Lo anterior se transformó en una tendencia para la OFT hasta su cierre.

De hecho, tras las modificaciones del régimen de protección del consumidor introducidas en 2013, los Local Authority Trading Standards Services comenzaron a desempeñar un papel más importante en la aplicación de la legislación de protección del consumidor a nivel nacional y la OFT inclinó su actuar a violaciones que demostrasen fallas sistémicas en un mercado. Es decir, sus acciones ya no se dirigían en contra de empresas individuales, sino a grupos de varias empresas o sectores, a menos que lo primero fuese conveniente para sentar precedente o pudiese tener impacto en el mercado. ${ }^{51}$

\footnotetext{
48 The Office of Fair Trading v Ashbourne Management Services Ltd EOOrs [2011] EWHC $1237($ Ch).

49 Para ver las implicancias del caso Ashbourne: CMA. Health and fitness clubs: unfair contract terms. Office of Fair Trading (OFT) closed consumer enforcement case. Disponible en: https://wrwre.gov.uk/cma-cases/health-and-fitness-clubs-unfair-contract-terms, consultado el 17 de enero de 2017.

50 Se trata de denuncias, realizadas por organismos protectores de los derechos de los consumidores, relativas a un mercado que está, o parece estar, perjudicando significativamente los intereses de los consumidores (Enterprise Act, 2002: Section 11).

51 Disponible en: http://webarchive.nationalarchives.gov.uk/20140525130048/http://wrere. oft.gov.uk/OFTwork/consumer-enforcement/internet-enforcement/;jsessionid=EC4FCAA88551E13C B267FB12B074179F, consultado el 21 d enero de 2017.
}

Esta obra está bajo una Licencia Creative Commons Atribución-NoComercial-SinDerivar 4.0 Internacional, IIJ-UNAM. Boletín Mexicano de Derecho Comparado, núm. 154, enero-abril de 2019, pp. 171-198. 
Para que quede más claro es útil una comparación de compromisos adoptados en distintos periodos: entre abril de 2003 y marzo de 2004 fueron 116 (OFT, Anual Report, Summary of Oft Consumer Law Casework, 2004), y entre abril de 2012 y marzo de 2013, la OFT había concluido sólo 20 (OFT, Consumer protection casework, 2013).

Lo que se buscaba eran cambios en las condiciones generales aplicables a gran parte de un sector, lo cual maximizó el impacto de su trabajo. Concretamente se negociaba con las organizaciones de proveedores que representaban sectores con importantes problemas de cláusulas abusivas, proveyéndolos de modelos de contratos de aplicación general. Con esta estrategia se lograba influir preventivamente sobre una mayor cantidad de proveedores, negociando de manera individual con cada uno de ellos (OFT, Anual Report and Resource Accounts, 2004: 30).

Por ejemplo, en el mercado de los autos usados, se logró que alrededor de 300 concesionarios utilizaran las condiciones general resultantes de la acción de la OFT (Anual Report and Resource Accounts, 2004: 30). En el sector de las ticketeras, se lograron condiciones más justas para los consumidores como resultado de una negociación con la Society of Ticket Agents and Retailers (STAR), donde varios de sus miembros, entre ellos Lastminute. com, Ticketmaster y the Big Bus Company, estuvieron de acuerdo en implementar un modelo de contrato revisado por la OFT (Annual Report and Resource Accounts, 2009-2010: 26).

Para potenciar el impacto de la prevención del uso de cláusulas abusivas, dentro de este enfoque sectorial que se viene comentando, la OFT publicaba guías u orientaciones (guidance) (National Audit Office, 2003: 31-33) para sectores comerciales específicos considerados especialmente riesgosos para los intereses de los consumidores, utilizando como criterio el número de denuncias, por ejemplo: "Guidance on unfair terms in tenancy agreement" ${ }^{52}$ " "Guidance on unfair terms in health and fitness club agreements". ${ }^{33}$

La OFT ha entendido que los consumidores que comprenden sus derechos desempeñan un papel importante en asegurar que las empresas cumplan con el derecho del consumo, por dicha razón buscó alinear la

52 Disponible en: https://wwrw.gov.uk/government/publications/unfair-terms-in-tenancy-agreements-2, consultado el 17 de enero de 2017.

53 Disponible en: https://wrere.gov.uk/government/publications/unfair-terms-in-health-andfitness-club-agreements, consultado el 17 de enero de 2017.

Esta obra está bajo una Licencia Creative Commons

Atribución-NoComercial-SinDerivar 4.0 Internacional, IIJ-UNAM.

Boletín Mexicano de Derecho Comparado, núm. 154, enero-abril de 2019, pp. 171-198. 
educación de los consumidores con la de los proveedores para maximizar el impacto a través de publicaciones periódicas de guías y reportes. Es más probable que las empresas mejoren su cumplimiento de la norma si perciben que los consumidores estarán más capacitados (OFT, Consumer Law and Business Practice Drivers, 2010: 63-64).

\section{CONSIDERACIONES FINALES}

El control preventivo de cláusulas abusivas ha existido en el Reino Unido desde 1994. Desde entonces, el trabajo de la OFT ha evolucionado desde la revisión de una gran cantidad de casos involucrando a empresas individualmente consideradas hacia un enfoque sectorial basado en la revisión de condiciones generales de los contratos por sectores, maximizando el impacto de su actuación.

Dentro de esa evolución hay ciertas características en la forma de ejercer el control que se mantuvo y que es, seguramente, la razón del éxito.

Lo primero es el modelo de enforcement, que prefiere el cumplimiento ante la sanción (compliance approach) en el contexto de una pirámide dinámica donde la negociación (o incluso la autorregulación) se encuentran en la base y la sanción en la cúspide (pyramid of enforcement).

El trabajo de la OFT grafica muy bien lo anterior. Tal fue la preeminencia que se le dio a la negociación que el organismo ha sido calificado como "un agente negociador para los consumidores" (Ramsay, 2012: 357). Con ese enfoque, la OFT era capaz de lograr el cumplimiento voluntario a través de negociaciones informales o, a partir de compromisos con los proveedores, aplicando un mecanismo de control administrativo mixto que combina la actuación de la agencia con la voluntad de las empresas.

Este órgano administrativo influía, además, en el cumplimiento voluntario de la norma, participando de la elaboración de códigos de buenas prácticas que, a su vez, fomentaban la existencia de mecanismos de ADR; publicando detalladamente los casos revisados y las cláusulas consideradas abusivas para informar a consumidores, empresarios y demás enforcers; y, publicando guías u orientaciones sobre cláusulas abusivas por sector.

Eso no era todo. Además, la OFT tenía el deber de monitorear el cumplimiento de los acuerdos o compromisos referidos a la abstención del uso de las cláusulas consideradas abusivas, si la negociación fracasaba o si el compromiso no se cumplía, entonces la agencia accionaba ante los 
tribunales para obtener la declaración de abusividad, llegando así a activar el control judicial.

Como sostiene Bright (2000), es interesante especular por qué esta fórmula mixta de control ha tenido tanto éxito y por qué los proveedores han abandonado o modificado cláusulas generales, sin forzar el asunto hasta llegar al control judicial. Según la autora, presumiblemente, por el miedo a la mala publicidad. También está el hecho de que muchas de las cláusulas pueden ser consideradas injustas sólo en parte, entonces modificándolas se puede salvar parte de su redacción original, en cambio bajo el control judicial se declara toda la cláusula como abusiva y no obligará a los consumidores.

Se podría agregar a lo anterior algún interés por parte de los sectores empresariales de evitar los efectos de la doctrina del precedente, ya que una vez declarada la abusividad de una cláusula, muy probablemente influirá en la modificación, a instancias de la agencia estatal, de todos aquellos contratos que contengan cláusulas similares, sea voluntariamente, por persuasión, por negociación, vía undertaking, o por sentencia judicial.

En síntesis, el control preventivo administrativo de cláusulas abusivas del derecho inglés puede ser catalogado como experiencia que se debería mirar como objeto de microcomparación cuando se busca mejorar el respectivo sistema interno. Las razones se resumen a continuación.

Primero, porque reúne varios mecanismos de control: voluntario, administrativo y judicial, atacando a las cláusulas abusivas desde varios flancos, evitando la permeabilidad del sistema.

Segundo, porque el control se ejerce mayormente sobre cláusulas generales de la contratación (UTCCR, 1999: Regulation 12) y no sobre contratos de adhesión en particular. Además, se intenta abarcar sectores del mercado al ejercer control a través de asociaciones de proveedores o siguiendo casos con un alto impacto. Ambas circunstancias maximizan los efectos del control, logrando alcanzar una gran cantidad de potenciales contratos.

Tercero, porque el ejercicio del control administrativo mixto y la activación del control judicial se entrega a varios organismos coordinados por la OFT, lo que refuerza o fortalece el control, sobre todo cuando se trata de materias cuya especialidad requiere de cierta expertiz, como el sector de las telecomunicaciones, por ejemplo. 
Cuarto, se evita la sanción buscando el cumplimiento de la norma jurídica.

Por último, si se piensa implementar un trasplante de este modelo a un sistema perteneciente a la familia del civil law, el hecho de que el derecho inglés pertenezca a otra familia jurídica no debiera ser obstáculo, toda vez que, como ya se explicó, el derecho del consumo en el Reino Unido se encuentra contenido, especialmente, en leyes.

Se debe tener en cuenta, además, que este sistema o modelo de control preventivo ha sido muy bien valorado a lo largo de su vigencia. Se pueden citar varias opiniones autorizadas, tanto de doctrina como informes de organismos que resaltan su buen funcionamiento. Por ejemplo, Bright ha sostenido que se trata de un sistema particularmente eficaz (Bright, 2000: 332); por su parte la National Audit Office ha destacado el éxito del sistema y su eficiencia; ${ }^{54}$ según la Comisión Europea el sistema de control de cláusulas abusivas del Reino Unido ha traído resultados bastante satisfactorios; ${ }^{* 5}$ Ramsay ha señalado que la experiencia de la OFT evidencia la capacidad de una agencia administrativa para producir cambios en las condiciones generales de un sector; ${ }^{56}$ y Collins, comentando un trabajo de Alpa, sostiene que el trabajo de la OFT parece haber sido especialmente eficaz controlando las cláusulas abusivas y que dicho éxito tiene relación justamente con el enfoque de la agencia que favorece la negociación (Micklitz, 2008: 10).

Las anteriores afirmaciones permiten sostener que el sistema inglés de control preventivo de cláusulas abusivas, conformado de una fórmula mixta y coordinada de mecanismos de control, sin perjuicio del control judicial

54 "La persuasión ha sido exitosa: los consumidores se beneficiaron con más de 100 millones de libras esterlinas del trabajo de la OFT en 1998 para combatir las cláusulas abusivas". "The OFT's action to combat unfair consumer contracts has secured savings for consumers at least fourteen times the cost of OFT's consumer protection work but greater savings are possible", Office of Fair Trading (1999: 7) (la traducción es propia).

55 "En su afán por conseguir la supresión efectiva de las cláusulas y lagunas abusivas, algunos sistemas nacionales de control de cláusulas abusivas (como el Ombudsman en los países nórdicos o la Office of Fair Trading en el Reino Unido) han favorecido la negociación directa con los profesionales individuales y las asociaciones profesionales, lo que ha permitido obtener resultados halagüeños" (Comisión de las Comunidades Europeas, 2000: 26).

56 "The experience of the OFT in implementing the UTCGR indicates the potential ability of an administrative agency to bring about broad-scale changes in industry terms (eg mobile phones)" (Ramsay, 2012: 330). 
represivo, ha logrado la protección de los derechos de los consumidores evitando la incorporación de cláusulas abusivas a un significativo número de contratos y es, por lo tanto, es un modelo de control preventivo ejemplar.

\section{BiBLIOGRAFÍA}

ALPA, Guido, 2008, "Standard Contract Terms: the Role of the Courts and Moral Persuasion by Independent Authorities", en Collins, H. (ed.), Standard Contract Terms in Europe. A Basis for and a Challenge to European Contract Law, Países Bajos, Kluwer Law Internationals BV.

Appenzzeller, Carmen, 2017, "Towards a More Effective Regulation of Unfair Standard Contract Terms in Europe: of Cartels, Watchdogs and a "Gorilla in the Closet»", EuCML.

BRIGHT, Susan, 2000, "Winning the Battle against Unfair Contract Terms", Legal Studies.

CotTerrell, Roger, 2006, "Comparative Law and Legal Culture", The Oxford Handbook of Comparative Law, Oxford, Oxford University Press.

Cseres, Katalin, 2005, Competition Law and Consumer Protection, Países Bajos, Kluwer Law International.

DAVID, René, 1980, English Law and French Law: A Comparison in Substance, London, Stevens \& Sons and Calcutta, Eastern Law House.

GRAZIEDEI, Michele, "Comparative Law as the Study of Transplants and Receptions", en Reimann, R. y Zimmermann, M. (eds.), The Oxford Handbook of Comparative Law, Oxford, Oxford University Press.

Howells, Geraint y Weatherill, Stephen, 2005, Consumer Protection Law, 2a. ed., Aldershot, Ashgate.

Libchaber, R., 1997, "L'autorité des recommandations de la Commission des clauses abusive", RTD Civ.

MANCERA, Adrián, 2008, "Consideraciones durante el proceso comparativo", Boletín Mexicano de Derecho Comparado.

MAZA, Íñigo de la, 2012, "El control de las cláusulas abusivas y la letra G", en BARrientos, C. et al. (eds.), Consumidores, Legal Publishing Chile.

MaCDONALD, Elizabeth, 2002, "Scope and Fairness of the Unfair Terms in Consumer Contracts Regulations, Director General of Fair-Trading v First National Bank", Modern Law Review, vol. 65.

Micklitz, Hans, 2005, The Politics of Fudicial Co-Operation in the EU. Sunday Trading, Equal Treatment and Good Faith, Cambridge University Press.

Esta obra está bajo una Licencia Creative Commons

Atribución-NoComercial-SinDerivar 4.0 Internacional, IIJ-UNAM.

Boletín Mexicano de Derecho Comparado, núm. 154, enero-abril de 2019, pp. 171-198. 
Micklitz, Hans, 2008, "Some Reflections on Cassis de Dijon and the Control of Unfair Terms in Consumer Contracts", en Collins, H. (ed.), Standard Contract Terms in Europe. A Basis for and a Challenge to European Contract Law, Países Bajos, Kluwer Law International.

Morales, María Elisa, 2017, "Reforma al derecho inglés en materia de cláusulas abusivas", Revista Chilena de Derecho Privado.

NAVA, Wendolyne, 2017, "Problemas de ejecución en el marco de los sistemas extrajudiciales de resolución de conflictos en línea, vinculados al comercio electrónico B2C", Boletín Mexicano de Derecho Comparado.

NigLIA, Leone, 2003, The Transformation of Contract in Europe, Países Bajos, Kluwer Law International.

RAMSAY, Iain, 2012, Consumer Law and Policy. Text and Materials on Regulating Consumer Markets, 3a. ed., Portland, Oregon, Oxford y Portland, Hart Publishing.

Riefa, Christine y WiLlett, Chris, 2018, "Enforcement and Effectiveness of Consumer Law in the UK", en Micklitz, Hans y SAUMIER, Geneviève (eds.), Enforcement and Effectiveness of Consumer Law, Springer, Gewerbestrasse.

WoOdroffe, Geoffrey y LOWE, Robert, 2013, Woodroffe and Lowe's Consumer Law and Practice, 9a. ed., Londres, Sweet y Maxwell.

ZweIGERT, Konrad y KöTZ, Hein, 1998, Introduction to Comparative Law, Oxford, Clarendom Press.

\section{Otros documentos}

Consejo de la Unión Europea, 1993, Directiva 93/13/CEE del Consejo, del 5 de abril, sobre las cláusulas abusivas en los contratos celebrados con consumidores, 16 de abril de 1993, DOUE, 21 de abril de 1993.

Comisión de las Comunidades Europeas, 2000, Informe de la Comisión sobre la Aplicación de la Directiva 93/13/CEE del Consejo, del 5 de abril de 1993, sobre las cláusulas abusivas en los contratos celebrados con consumidores, Bruselas, 27 de abril de 2000.

LONDON ECONOMICS, 2009, Evaluation of a sample of Consumer Enforcement Cases. Disponible en: http://londoneconomics.co.uk/wp-content/ uploads/2011/09/32-Evaluation-of-a-sample-of-OFTs-consumer-enforcementcases.pdf, consultado el 9 de agosto de 2015. 
Esta revista forma parte del acervo de la Biblioteca Jurídica Virtual del Instituto de Investigaciones Jurídicas de la UNAM

National Audit OfFice, 2003, The Office of Fair Trading Progress in Protecting Consumers' Interests. Report by the Comptroller and Auditor General, Hc 430 Session 2002-2003, 6 de marzo de 2003.

Office of FAIR Trading, 2004, Anual Report and Resource Accounts 20032004, Objective 1: Enforcing Consumer Protection Legislation.

OfFice of FAIR Trading, 2004, Anual Report and Resource Accounts 20032004, Summary of Oft Consumer Law Casework 2003 to 2004 Excluding Consumer Credit.

OfFice of Fair Trading, 1996, Unfair Contract Terms Bulletin, núm. 1.

OfFice OF Fair Trading, 1999, Protecting the Consumer from Unfair Trading Practices.

Office of Fair Trading, 2010, Consumer Law and Business Practice Drivers of Compliance and Non-Compliance.

OfFice OF FAIR Trading, 2013, Consumer protection casework, 1o. de abril de 2012 al 31 de marzo de 2013.

Office of Fair Trading, 2014, Annual Report and Accounts 2013 to 2014.

Parlamento Del Reino Unido, 2002, Enterprise Act, 7 de noviembre de 2002, sobre los cinco objetivos principales de política de competencia.

Parlamento Del Reino Unido, 2013, Enterprise and Regulatory Reform Act, 25 de abril de 2013.

EuroPe DiREGT, Case_ID: 1034290/4058184, pregunta sobre Directiva 93/13.

Parlamento del ReIno Unido, 1994, The Unfair Terms in Consumer Contracts Regulations (UTCCR), Consumer Protection, núm. 2083, vigente a partir del 1o. de julio de 1995.

Parlamento del Reino Unido, 1999, The Unfair Terms in Consumer Contracts Regulations, Regulation 15, núm. 2083, vigente a partir del 1o. de octubre de 1999.

Scottish LaW Commission, Unfair Terms in Consumer ConTRACTS: A New APPROACH?, Summary of the Issues Paper, 25 de julio de 2012. 